\title{
Synchronous Multiple Primary Lung Cancer: A Case Report
}

\author{
Alexei L. Charyshkin, PhD, ScD ${ }^{1 *}$; Evgeniy A. Toneev ${ }^{1,2}$; Alexander A. Martynov²; \\ Ruslan I. Lisyutin²; Airat Sh. Zul'karnyaev² \\ ${ }^{1}$ Ulyanovsk State University, ${ }^{2}$ Ulyanovsk Regional Oncology Center \\ Ulyanovsk, the Russian Federation
}

\begin{abstract}
The incidence of multiple primary malignancies has increased dramatically in recent decades. Herein we report synchronous multiple primary lung cancer (SMPLC) in a 52-year-old male patient. It is advisable to consider SMPLC as a local pathology and to perform radical surgical treatment, which contributes to a favorable outcome. (International Journal of Biomedicine. 2018;8(2):162-164.)
\end{abstract}

Key Words: synchronous multiple primary lung cancer $\bullet$ multiple primary malignancies $\bullet$ pneumonectomy $\bullet$ lymph node

\section{Abbreviations}

LN, lymph node; MPC, multiple primary cancer; MPLC, multiple primary lung cancer; SMPLC, synchronous MPLC.

\section{Introduction}

Multiple primary cancer (MPC) was defined by Warren and Gates as 2 or more tumors occurring at different locations that are histologically malignant and distinct, such that one tumor is not a metastasis of the other. ${ }^{(1)}$ Based on the time when the tumors are identified, the disease can be classified as synchronous (MPC diagnosed within six months of the primary cancer diagnosis) or metachronous (MPC diagnosed more than six months after primary cancer diagnosis)..$^{(2-4)}$

An analysis of several studies showed that the incidence of multiple primary malignancies is estimated in $0.73 \%-5.2 \%$ of all tumor patients. ${ }^{(5)}$ In Russia, this rate was 7.5\% (in 2016) of patients with all malignant neoplasms. ${ }^{(6)}$ The incidence of MPLC has been reported to range from $0.7 \%$ to $15 \%$ of patients with lung cancer. ${ }^{(7-11)}$

Diagnosis of MPLC is complicated by difficulties in distinguishing it from lung cancer metastasis. SMPLC is characterized by the presence of the second tumor

*Corresponding author: Prof. Alexei L. Charyshkin, PhD, $S c D$, Head of the Faculty Surgery Department, Institute of Medicine, Ecology and Physical Education, Ulyanovsk State University. Ulyanovsk, the Russian Federation.E-mail: charyshkin@yandex.ru concurrently. The incidence of SMPLC has been variably reported as being between $1 \%$ and $16 \%{ }^{(7)}$ Distinguishing intrapulmonary metastatic tumors from MPLC is difficult but of great importance for the therapeutic management and prognosis of these patients. ${ }^{(12-14)}$

\section{Case presentation}

A 52-year-old man was admitted to our institution for the planned surgical treatment on June 16, 2017. The patient's medical history showed that shortness of breath while exercising and cough first appeared in April 2017. After visiting the district therapist, during the $\mathrm{X}$-ray examination a tumor of the right lung was detected. The patient was referred to an oncological dispensary for a consultation with a thoracic surgeon, where, after a post-examination, lung cancer of the right middle lobe was identified.

Fibrobronchoscopy (06.09.2017): the glottis was passable freely, the trachea and the main bronchi without pathology, the carina was mobile. The left lung was examined up to subsegmental bronchi, pathology was not revealed. Right lung: an infiltration of the mucosa at the mouth of S5; a biopsy of the lesion was performed for a histological and cytological examination. The remaining bronchi were passable, without pathology. 
The contrast-enhanced CT examination of the chest (05.22.2017): a right middle lobe infiltrate $(76 \times 53 \mathrm{~mm}$ in size), atelectasis (Fig.1); a focal pulmonary lesion (subpleural) in $\mathrm{S} 3$ of the right lung $(9.4 \times 6.8 \mathrm{~mm}$ in size $)$, the accumulation of contrast was impossible to reliably determine (Fig.2). The pulmonary hilar LNs were enlarged up to $10-14 \mathrm{~mm}$.

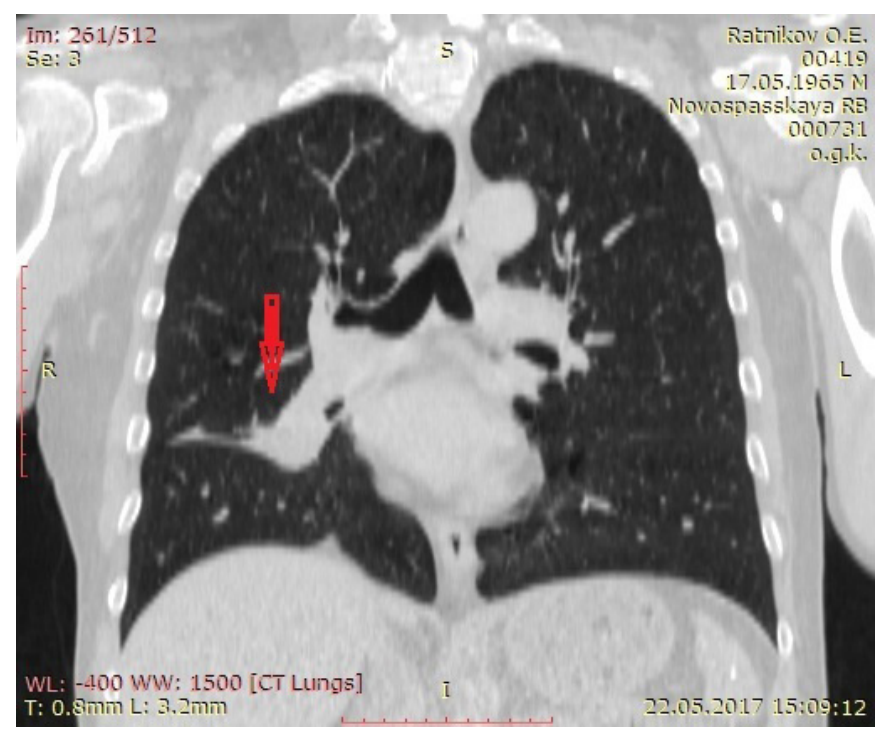

Fig.1. A right middle lobe infiltrate $(76 \times 53 \mathrm{~mm}$ in size $)$.

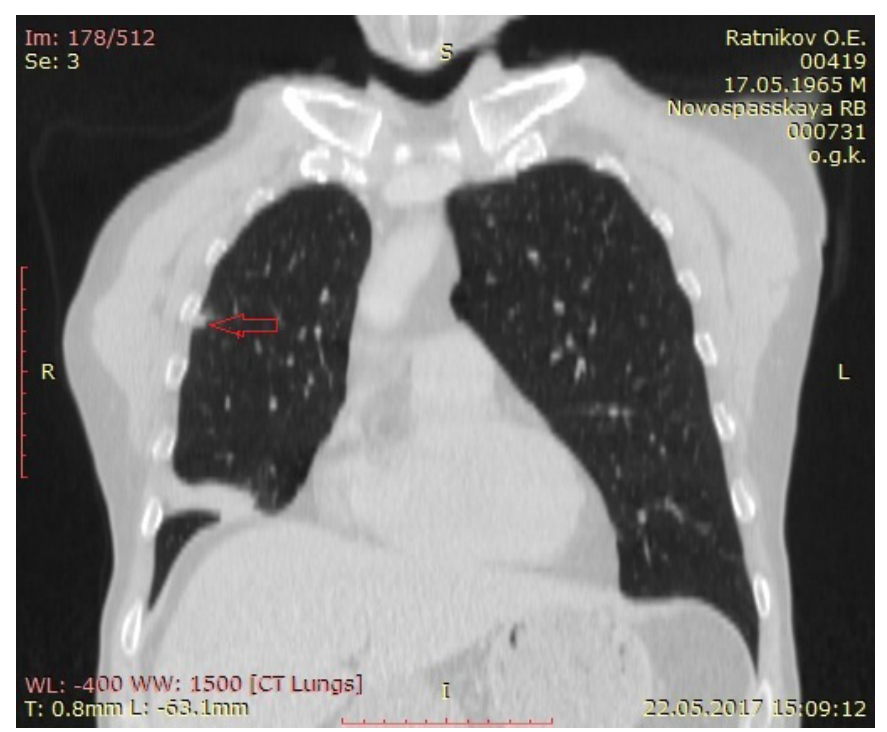

Fig.2. A focal pulmonary lesion (subpleural) in S3 of the right lung $(9.4 \times 6.8 \mathrm{~mm}$ in size $)$.

Abdominal ultrasound (05.29.2017): the abdominal cavity and retroperitoneal space without pathology. Spirography: FEV1 - 87\%, VC - 90\%. The Echo and ECG data are within normal limits. Clinical blood tests are within the norm.

Objective data: the patient had a satisfactory state, clear consciousness, and preserved intellectual function; he was oriented, critical and emotionally labile. Weight - $84.5 \mathrm{~kg}$, height $-184 \mathrm{~cm}$. The patient was of normosthenic constitution.
Skin cover was smooth and normal color. The leg veins were dilated in the system of the great saphenous vein. Peripheral LNs were not palpable. Thyroid gland is not enlarged. He had a bronchial breath sound in the right upper lobe; respiratory rate was 18 breaths per minute. Cardiovascular examination revealed normal heart sounds, blood pressure $-120 / 80 \mathrm{mmHg}$. The liver was enlarged, painful on palpation.

Based on clinical and anamnestic results of the examination, the following clinical diagnosis was constructed: Lung cancer of the right middle lobe (T2bNxM0). Histological diagnosis: moderately differentiated squamous cell carcinoma.

On June 19, 2017, given the clinical diagnosis, the planned operation was performed: Right thoracotomy, pneumonectomy with systematic lymph node dissection.

\section{Intraoperative}

Under endotracheal anesthesia, a right anterolateral thoracotomy performed in the fifth intercostal space revealed a $3 \times 4 \mathrm{~cm}$-sized nodule in S5 of the right middle lobe (tumor $1)$, atelectasis of the middle lobe. In the upper lobe in S3, the focus of $1 \times 1 \mathrm{~cm}$ (tumor 2 ) with invasion into the pleura, a conglomerate of bifurcation LNs up to $15 \mathrm{~mm}$ in size, single lymph nodes of the pulmonary ligament. Other groups of LNs are not enlarged. An operation was possible in the form of pneumonectomy. Next, a standard right pneumonectomy with systemic ipsilateral lymph node dissection was performed. There were no intraoperative complications. The postoperative period proceeded smoothly.

\section{Histological examination}

In the upper lobe of the right lung: well differentiated adenocarcinoma of a mixed structure (acinar-tubularpapillary) with invasion into the visceral pleura. In the middle lobe: carcinosarcoma. A line of resection of the bronchus without tumor growth. In one peribronchial LN (out of 17), there was metastasis of papillary adenocarcinoma (i.e., metastasis of tumor 2). In one bifurcation LN (out of 7), there was metastasis of carcinosarcoma (i.e., metastasis of tumor 1). The remaining separately sent LNs (2 pulmonary ligament LNs, azygos lymph node, 6 LNs of paratracheal group) were without metastases.

\section{Immunohistochemical examination}

Tumor 1: EMA, vimentin - diffuse bright expression; CK7, TTF-1 - diffuse bright expression in the epithelial component and focal moderate expression in sarcomatous component. CK5/6 moderate diffuse expression in the epithelial component. p63, SMA, CD34-negative. S100-focal moderate expression. CD68 - marked expression. Desmin — diffuse bright expression in the epithelial component. Histological diagnosis: primary lowgrade adenocarcinoma of the lung with sarcomatous component and focal squamous differentiation.(Fig.3)

Tumor 2: TTF-1—diffuse expression, which confirms a primary character of the tumor. Histological diagnosis: Primary well differentiated adenocarcinoma. (Fig.4)

The final clinical diagnosis. SMPLC: 1. Lung cancer of the right middle lobe (pT2aN2M0). Primary low-grade adenocarcinoma with sarcomatous component and focal squamous differentiation. 2. Lung cancer of the right upper lobe (pT1aN1M0). Primary well-differentiated adenocarcinoma. Three metastatic LNs: one LN of pulmonary root and two mediastinal LNs. 


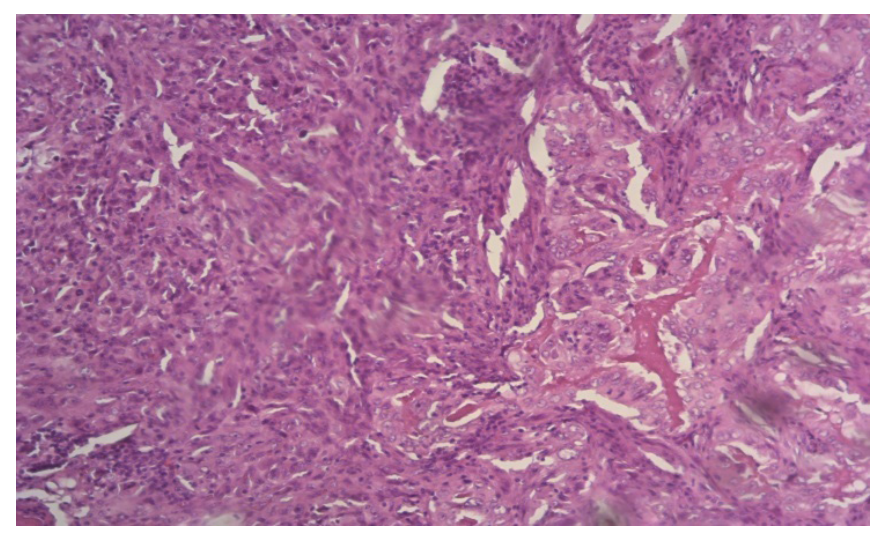

Fig. 3. Tumor 1. Immunohistochemical examination: EMA, vimentindiffuse bright expression; CK7, TTF-1-diffuse bright expression in the epithelial component and focal moderate expression in sarcomatous component.CK5/6- diffuse moderate expression in the epithelial component; p63, SMA, CD34-negative. S100-focal moderate expression. CD68-marked expression. Primary low-grade adenocarcinoma of the lung with sarcomatous component and focal squamous differentiation.

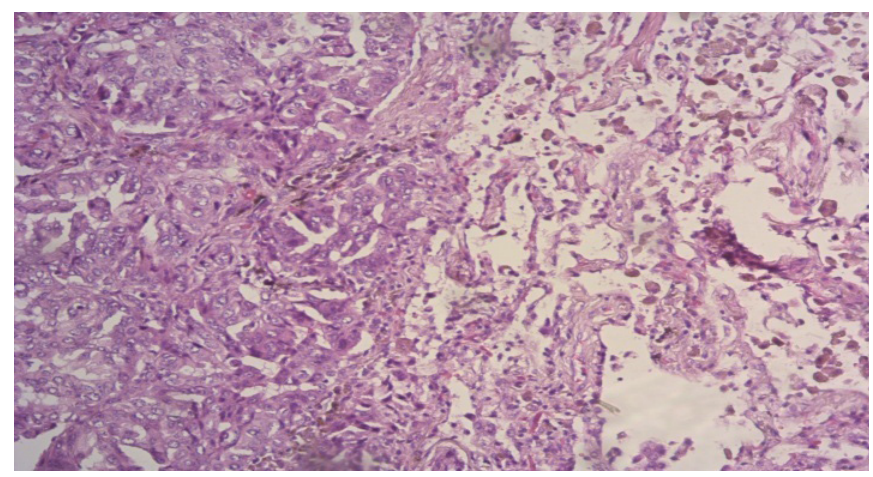

Fig. 4. Tumor 2. Immunohistochemical study:TTF-1-diffuse expression, which confirms a primary character of the tumor. Primary well differentiated adenocarcinoma.

The patient was discharged in a satisfactory condition on June 30, 2017. The patient received regular follow-up. After 3 weeks, adjuvant chemotherapy was started. Patient received 4 courses of adjuvant chemotherapy with cisplatin and etoposide. Patient had satisfactory response to treatment.

Follow-up of 10.30.2017: no active complaints. The contrast-enhanced CT examination of the brain, chest and abdominal organs did not reveal focal pathology. Fibrobronchoscopy: condition after right pneumonectomy, left lung without changes. The patient returned to his former work, socially adapted.

\section{Conclusion}

The presented clinical case proves the complexity of the diagnosis of polyneoplasias in one lung. In most cases, MPLC is an operational finding and is confirmed by histological examination. According to the recommendations of foreign clinical studies and this clinical example, it is advisable to consider SMPLC as a local pathology and to perform radical surgical treatment, which contributes to a favorable outcome.

\section{Competing interests} interests.

The authors declare that they have no competing

\section{Sources of Funding}

This study was funded by the Ministry of Education and Science of the Russian Federation (the science project No. 18.7236.2017/BCh).

\section{References}

1. Warren S, Gate O. Multiple primary malignant tumors. A survey of the literature and statistical study. Am J Cancer. 1932;16:1358-1414.

2. Grundmann RT, Meyer F. [Second primary malignancy among cancer survivors - epidemiology, prognosis and clinical relevance]. Zentralbl Chir. 2012;137(6):565-74. doi: 10.1055/ s-0031-1283939. [Article in German].

3. Feng FY, Zhang DC, Liu XY, Wang YG, Mao YS. [Surgical treatment and prognosis of synchronous double primary lung cancer: a report of 31 cases]. Ai Zheng. 2005;24(2):215-8. [Article in Chinese].

4. Pommier RF, Vetto JT, Lee JT, Johnston KM. Synchronous non-small cell lung cancers. Am J Surg. 1996;171(5):521-4.

5. Li F, Zhong WZ, Niu FY, Zhao N, Yang JJ, Yan HH, Wu YL. Multiple primary malignancies involving lung cancer. BMC Cancer. 2015;15:696. doi: 10.1186/s12885-015-1733-8 6. Kaprin AD, Starinsky VV, Petrova G.V. Status of oncological care for the population of Russia in 2016. M.:MNIOI named after P.A. Herzen; 2017.

7. Ferguson MK, DeMeester TR, DesLauriers J, Little AG, Piraux M, Golomb H. Diagnosis and management of synchronous lung cancers. J Thorac Cardiovasc Surg. 1985;89(3):378-85.

8. Verhagen AF, van de Wal HJ, Cox AL, Lacquet LK. Surgical treatment of multiple primary lung cancers. Thorac Cardiovasc Surg. 1989;37(2):107-11.

9. Lam S, MacAulay C, Palcic B. Detection and localization of early lung cancer by imaging techniques. Chest. 1993;103(1 Suppl):12S-14S.

10. Woolner LB, Fontana RS, Cortese DA, Sanderson DR, Bernatz PE, Payne WS, et al. Roentgenographically occult lung cancer: pathologic findings and frequency of multicentricity during a 10-year period. Mayo Clin Proc. 1984;59(7):453-66.

11. van Rens MT, Zanen P, Brutel de La Rivière A, Elbers HR, van Swieten HA, van Den Bosch JM. Survival in synchronous vs. single lung cancer: upstaging better reflects prognosis. Chest. 2000;118(4):952-8.

12. Finley DJ, Yoshizawa A, Travis W, Zhou Q, Seshan VE, Bains MS, et al. Predictors of outcomes after surgical treatment of synchronous primary lung cancers. J Thorac Oncol. 2010;5(2):197-205. doi: 10.1097/JTO.0b013e3181c814c5.

13. Powell S, Tarchand G, Rector T, Klein M. Synchronous and metachronous malignancies: analysis of the Minneapolis Veterans Affairs (VA) tumor registry. Cancer Causes Control. 2013;24(8):1565-73. doi: 10.1007/s10552-013-0233-X.

14. Shen C, Wang X, Tian L, Zhou Y, Chen D, Du H, et al. "Different trend" in multiple primary lung cancer and intrapulmonary metastasis. Eur J Med Res. 2015;20:17. doi: 10.1186/s40001-015-0109-5. 